\title{
INTERFERENCE BETWEEN THE PONTINE DETRUSOR NUCLEUS AND THE PONTINE URINE-STORAGE NUCLEUS
}

\author{
AN ELECTROMYOGRAPHICAL STUDY OF THE \\ EXTERNAL URETHRAL SPHINCTER
}

\author{
Y. Koyama, H. Ozaki And M. KurU \\ National Cancer Center, Tokyo, Japan
}

\begin{abstract}
In previous papers ${ }^{11,5)}$ two antagonistic centers concerning vesical function have been described, close to and quite separate from each other in the lateral reticular formation of the rostral pons. The one, the pontine detrusor nucleus (PDN), was identified with a part of locus coeruleus and the other, the pontine urine-storage nucleus (PUSN), was located in an area in the ventrolateral reticular formation directly dorsal to the most lateral part of the medial lemniscus and its vicinity in the rostral pons. Further, the related fibers connecting with each other and to the bulbar and sacral vesicomotor areas were described in detail ${ }^{11,5}$. These findings are diagramatically summarized in FIG. 1. These fiber connections imply the possibility of interference between these two centers, which may play an important role for the two vesical functions, urine storage and micturition. The present study was carried out in decerebrate cats to elucidate the nature of mutual interference between these pontine nuclei.
\end{abstract}

\section{MATERIAL AND METHODS}

Fifty adult cats weighing $2-3 \mathrm{~kg}$ were used. The animal was anesthetized with $25 \mathrm{mg} / \mathrm{kg}$ of nembutal administered intravenously; decerebrated supracollicularly and decerebellated to expose the floor of the fourth ventricle. After mid-line ventral incision, the symphysis pubis was cut off and the retropublic fat was removed by blunt dissection, so the urethra was exposed throughout its length. Then through a small incision at the bladder neck a thin catheter (Nélaton No. 4-5) was inserted into the bladder and fixed. The catheter, the electromanometer and the reservoir were connected with a T-cock. From the reservoir warm saline was introduced into the bladder at various rates. To observe the reflex contraction the reservoir was raised up gradually so that $20-40 \mathrm{ml}$ of saline was introduced in to the bladder in $30-60$ seconds. When the content reached this volume and bladder began to contract, the T-cock was

Received for publication November 30, 1965

小山靖夫, 尾崎秀雄, 久留 勝 

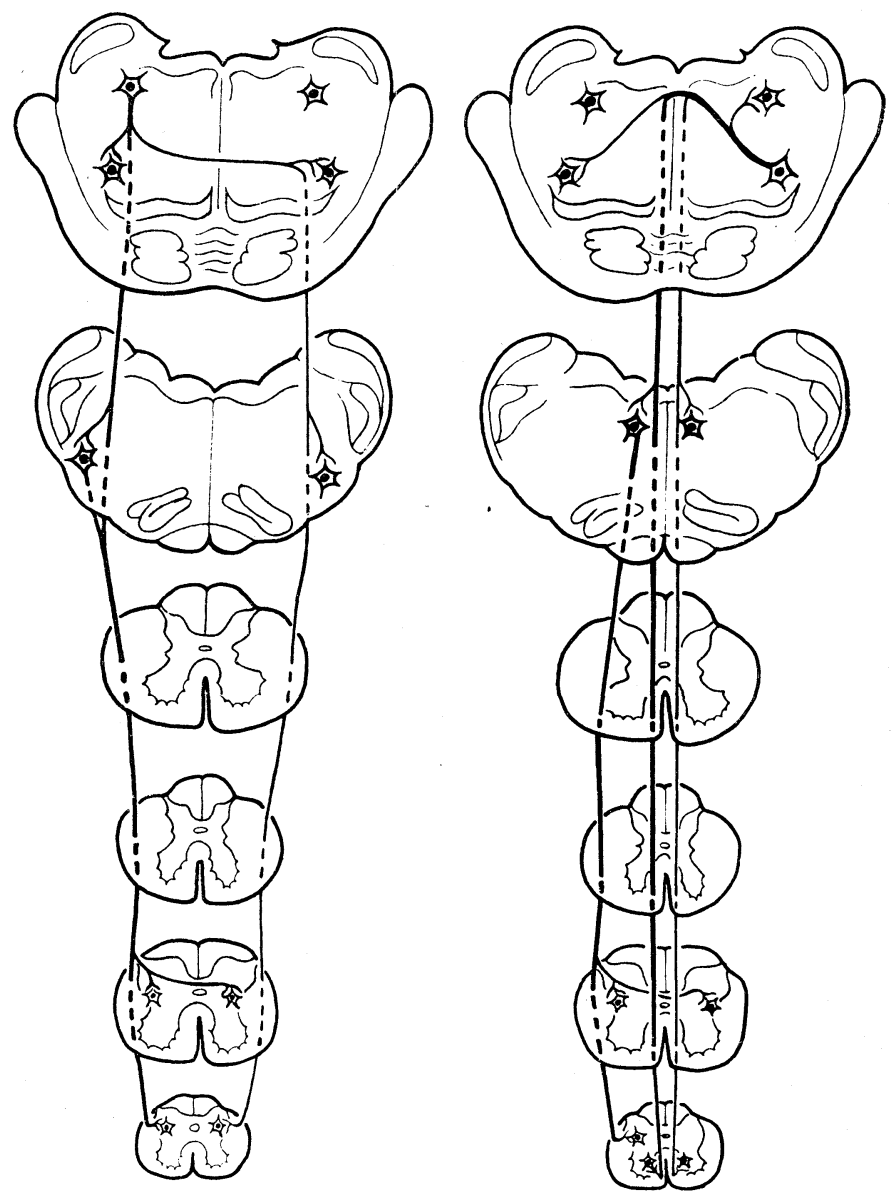

FIG. 1. Diagrams showing the origins, courses and terminations of three kinds of reticulo-spinal connections related to micturition. Upper two sections indicate rostral pons and mid-bulb; lower four sections indicate cervical, thoracic, lumbar and sacral segments, respectively. In the left diagram the lateral reticulo-spinal fibers originating from PDN (11) and from bulbar vesico-constrictor center (8) are shown and in the right diagram the ventral reticulo-spinal fibers originating from bulbar vesicorelaxer center (6) and the medial reticulo-spinal fibers originating from PUSN (5) are shown. Mesencephalic component of the lateral reticulospinal fibers (10) is omitted in this diagram.

closed to make the contraction "isovolumetric". Sometimes, for the purpose of quick distension, the reservoir was raised rapidly, (usually 50-100 $\mathrm{ml}$ was introduced in 10-20 seconds) and intravesical pressure reached $50-80 \mathrm{~cm} \mathrm{H}_{2} \mathrm{O}$, then the reservoir was lowered slowly to the previous level.

The distance between bladder and external urethral sphincter was $1.5 \mathrm{~cm}^{2}$ ). In the male cat a prominence ralated to the prostate was observed directly beneath the symphysis and the external urethral sphincter located at the distal part of the prostate. 
In female cats too, the external urethral sphincter was found at the corresponding part of the urethra, although it was less developed than in male cats. A steel electrode of $10-20 \mu$ tip diameter, insulated except the tip, was inserted into the external urethral sphincter. With a copper wire of $30 \mu$ in diameter the electrode was connected to the amplifier. The indifferent electrode was attached to the stump of the symphysis or to the subcutaneous tissue. The discharge from the sphincter and the pressure of the bladder were displayed simultaneously on a two-beam cathode-ray oscilloscope. The experimental arrangement is diagramatically shown in FIg. 2.

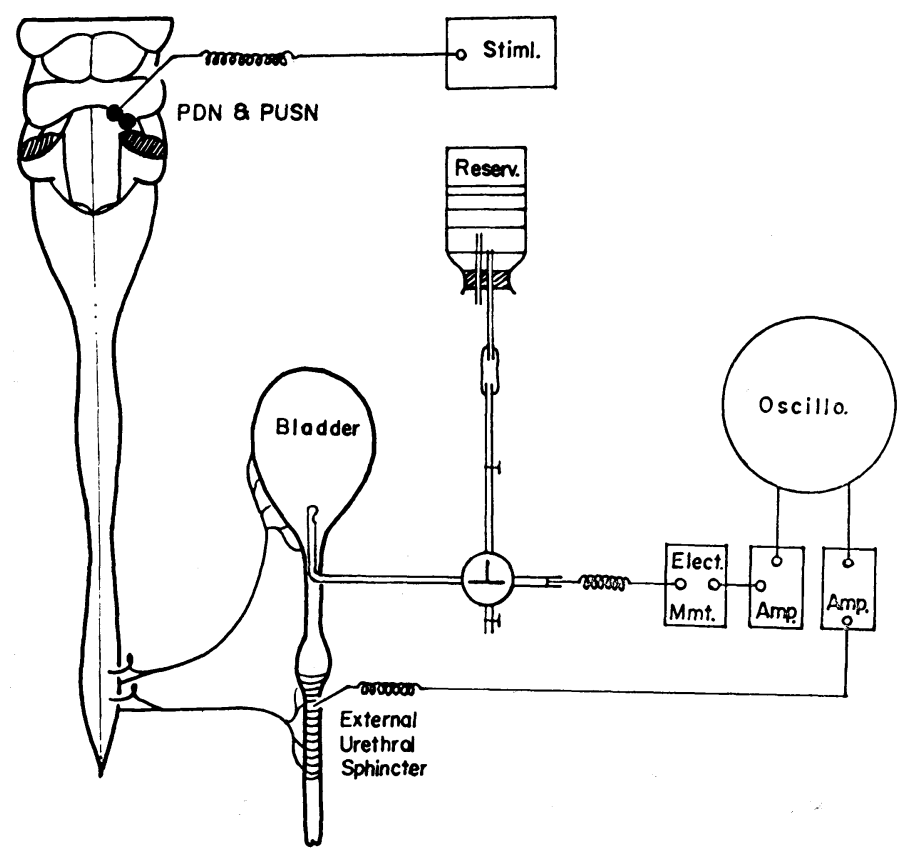

FIG. 2. Apparatus for demonstration of interference petween PDN and PUSN. For explanation see text.

In three animals experiments were performed after removal of the bladder. To remove the urinary bladder a lower abdominal incision was made, the urinary bladder was separated from the surrounding tissues, the distal portions of both ureters were ligated and cut and finally the bladder neck was ligated at its proximal end. Thus, the bladder was removed without injuring the external urethral sphincter and its nerve supply.

During the experiment the floor of the fourth ventricle and the sphincteric portion of the urethra were covered with liquid paraffin and temperature was maintained by infrared irradiation. In order to stimulate the PDN or PUSN, a unipolar needle electrode of $80 \mu$ tip diameter, insulated except the tip, was used. Electrode was inserted through the dorsal surface of the fourth ventricle. Stimulus was a rectangular pulse of $0.8-2.0 \mathrm{v}$ with the duration of $1 \mathrm{msec}$ at $50 \mathrm{c} / \mathrm{s}$. To check the location of stimulated point a minute electrical lesion was made by a direct current, $3 \mathrm{v}$ for 5 seconds, after the experiment. The brain stem was taken out and fixed. Celloidin serial section of $50 \mu$ thickness was prepared, stained with Weigert's method and the stimulated points were confirmed histologically. 


\section{RESULTS}

Change in sphincter discharge aue to the reflex contraction of the detrusor. When the detrusor was at rest, regular tonic discharges of relatively low frequency were recorded from the external urethral sphincter. When 20-40 ml of warm saline was dribbled into the empty bladder, rhythmical contractions of the bladder appeared after a certain lapse of time. Subsequently, undulation appeared in the intravesical pressure curve under the isovolumetric condition. Just prior to the rise of intravesical pressure, the discharge of the sphincter abruptly decreased and ceased entirely before the pressure curve attained the maximum. This abeyance of discharge retained as long as the bladder contraction continued. With the commencement of the bladder relaxation the sphincter activity reappeared, gradually increasing in discharge frequency, so that when the intravesical pressure returned to the previous level the discharge recovered the frequency before contraction (FIG. 3).

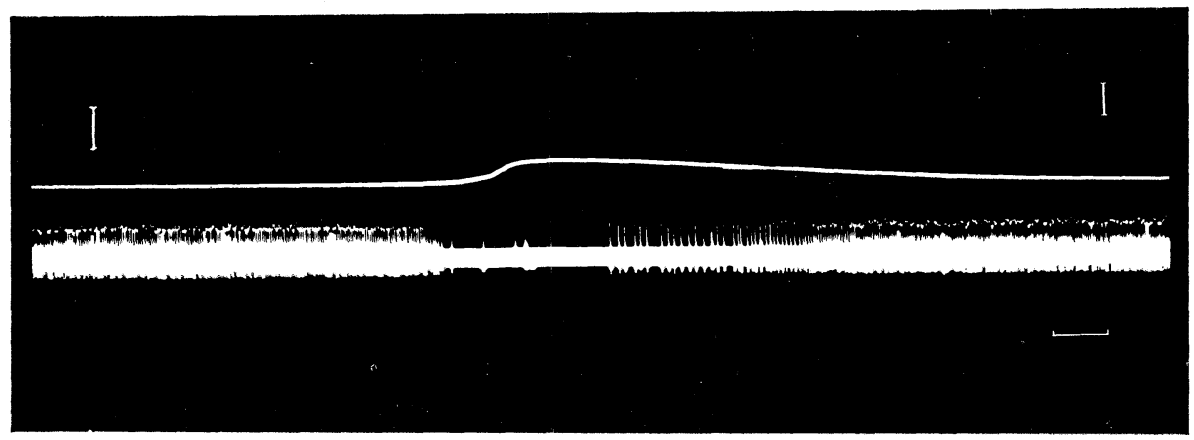

Fig. 3. Change of sphincter discharge due to reflex vesical contraction. Upper : intravesical pressure. Lower: electromyogram of the external urethral sphincter. Voltage calibration $200 \mu \mathrm{V}$ (vertical line on the right), scale for intravesical pressure $10 \mathrm{~cm} \mathrm{H}_{2} \mathrm{O}$ (vertical line on the left) and time scale $2 \mathrm{sec}$. (horizontal line on the lower right).

Sphincter response to PDN stimulation. Electrical stimulation to PDN provoked conspicuous vesical contraction. With the commencement of PDN stimulation, the discharge from sphincter was promptly inhibited and within 1-2 seconds it disappeared completely. During this period the intravesical pressure gradually rose and corresponding with the cessation of the sphincter activity the intravesical pressure rapidly rose and reached the maximum (20-50 $\left.\mathrm{cm} \mathrm{H}_{2} \mathrm{O}\right)$ usually within 5-10 seconds. During the stimulation the intravesical pressure remained at the maximum level and the discharge was completely suppressed. After the stimulation the sphincter activity promptly reappeared and gradually recovered the previous frequency, whereas the intravesical pressure slowly decreased to the initial level in $30-40$ seconds. 
The increase in the discharge frequency was intimately related to the decrease in intravesical pressure (FIG. 4). Occasionally marked rebound of the sphincter discharge was observed immediately after the cessation of stimulation. In this case, the increases in discharge frequency and amplitude were observed for several seconds after the cessation of stimulation. This kind of rebound was also sometimes observed in the detrusor.

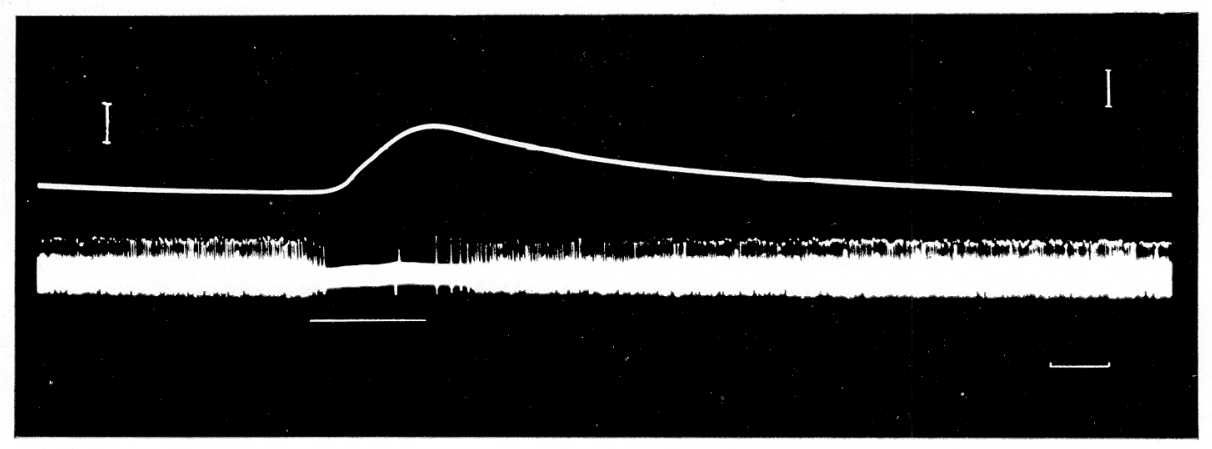

Fig. 4A. Responses of intravesical pressure (top) and sphincter discharge (middle) to the PDN stimulation (bottom). Voltage calibration $200 \mu \mathrm{V}$ (vertical line on the right), scale for intravesical pressure $10 \mathrm{~cm} \mathrm{H}_{2} \mathrm{O}$ (vertical line on the left) and time scale $2 \mathrm{sec}$. (horizontal line on the lower right).

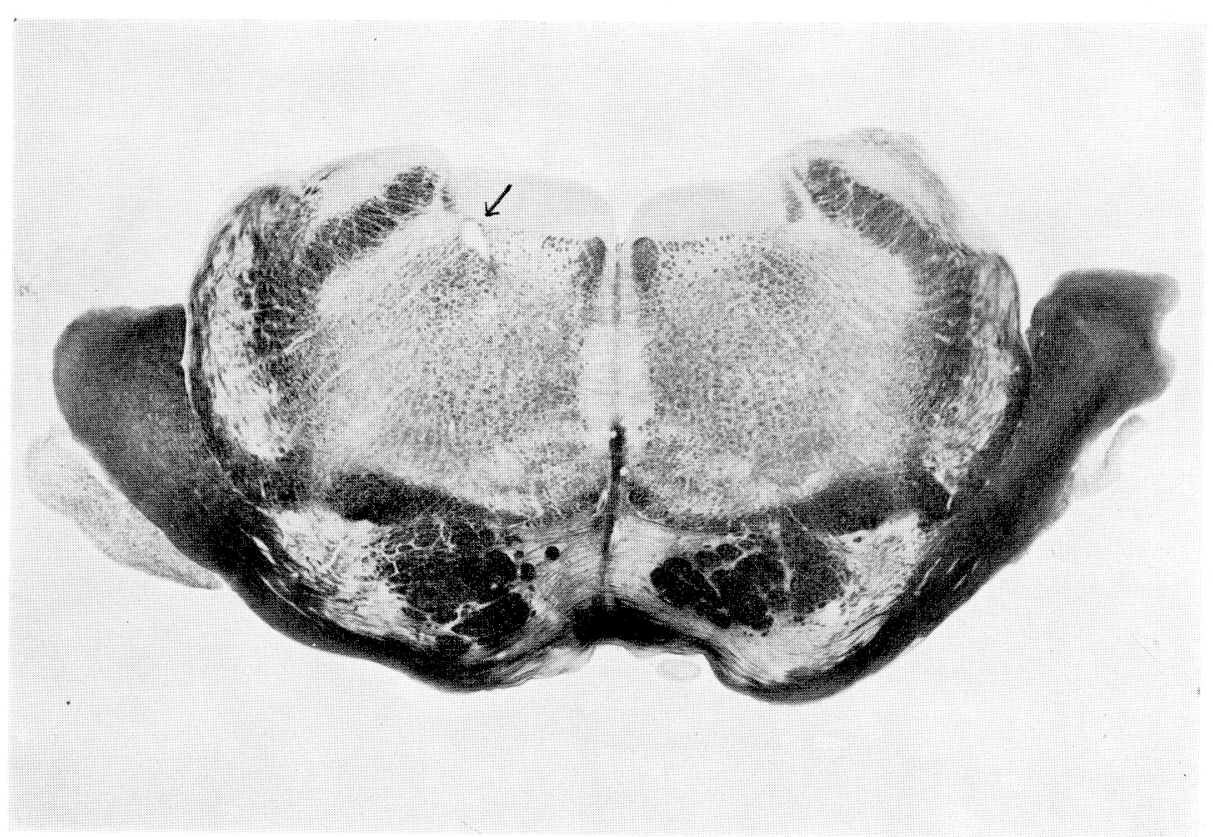

FIG. 4B. Electrolytic lesion made at the stimulated point of recording in FIG. $4 \mathrm{~A}$. Marked with arrow. 
Sphincter response to PUSN stimulation. In the resting bladder the stimulation to PUSN had no effect on the intravesical pressure. However, the discharge frequency of the external urethral sphincter was obviously increased during PUSN stimulation (FIG. 5A, left half).

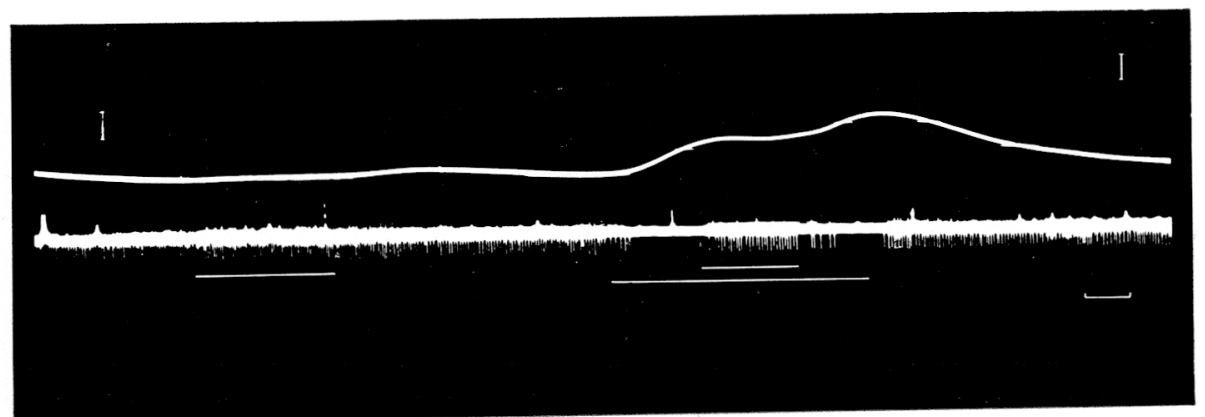

FIG. 5A. Responses of intravesical pressure and sphincter discharge to PUSN stimulation (left half of tracings), and to simultaneous stimulation of PDN and PUSN (right half of tracings). Tracings from above downward intravesical pres. sure, sphincter discharge, stimulation of PUSN and PDN respectively. Voltage calibration $200 \mu \mathrm{V}$ (vertical line on the right), scale for intravesical pressure $10 \mathrm{~cm}$ $\mathrm{H}_{2} \mathrm{O}$ (vertical line on the left) and time scale $2 \mathrm{sec}$. (horizontal line on the lower right).

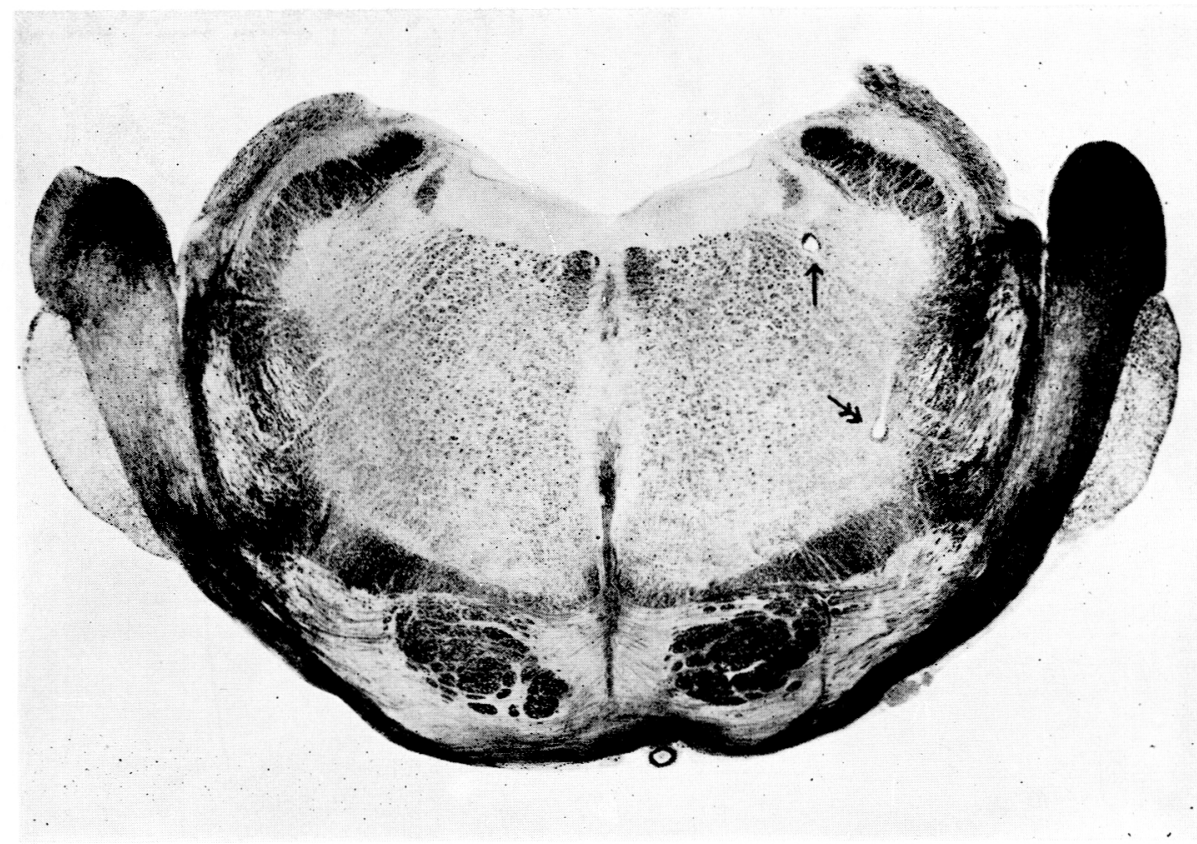

FIG. 5B. Electrolytic lesions made at stimulated points of recording in FIG. 5A. Indicated with single-winged arrow PDN and with double-winged arrow PUSN. 
Reaction of sphincter to combined stimulations of PDN and PUSN. Stimulating the PDN, the sphincter discharge disappeared and the intravesical pressure gradually rose. In addition the PUSN was stimulated, then the rate of rise of intravesical pressure stopped instantaneously, and simultaneously the sphincter discharge reappeared. When PUSN stimulation was stopped keeping PDN stimulation continued the sphincter activity disappeared again and the intravesical pressure gradually rose. After the stimulation to the PDN had been stopped, both the intravesical pressure and the sphincter activity gradually returned to their initial states, as observed in the second experiment (FIG. 5A, right half).

Response of the sphincter to a rapid distension of the bladder. When the vesical wall was distended by a rapid filling of the bladder with liquid, the sphincter discharge disappeared as GARRY et al. ${ }^{2)}$ already had remarked. In this case the decrease in sphincter discharge appeared later than the rise of the intravesical pressure. The discharge gradually decreased in frequency and finally disappeared completely. The rate of diminution of discharge frequency, however, was slower than that in the reflex vesical contraction. When the reservoir was lowered to evacuate the vesical contents, the sphincter activity gradually recovered to its resting level (FIG. 6). When the PUSN was stimulated after disappearance of sphincter discharge due to the rapid

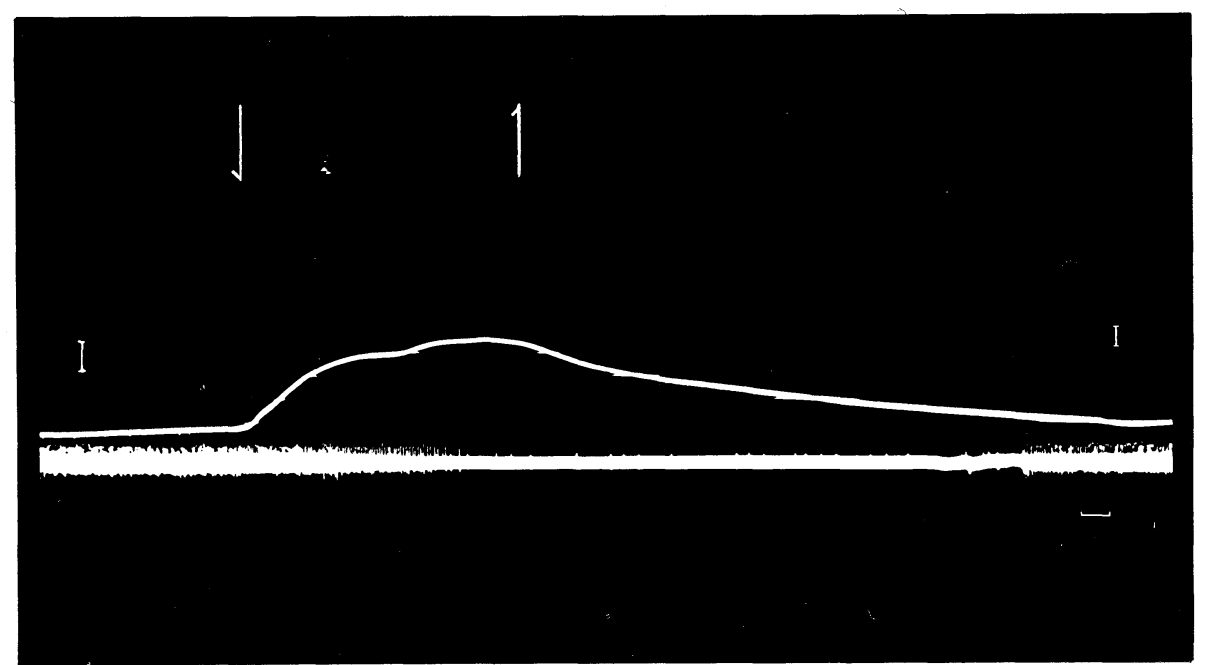

FIG. 6. Response of external urethral sphincter to passive distension of vesical wall following rapid filling of bladder. Upper: intravesical pressure. Vertical arrows on the top indicate onset $(\downarrow)$ and finish (1) of rapid filling of bladder respectively. Lower : discharge of external urethral sphincter. Voltage calibration $200 \mu \mathrm{V}$ (vertical line on the right), scale for intravesical pressure $10 \mathrm{~cm} \mathrm{H}_{2} \mathrm{O}$ (vertical line on the left) and time scale $2 \mathrm{sec}$, (horizontal line on the lower right). 


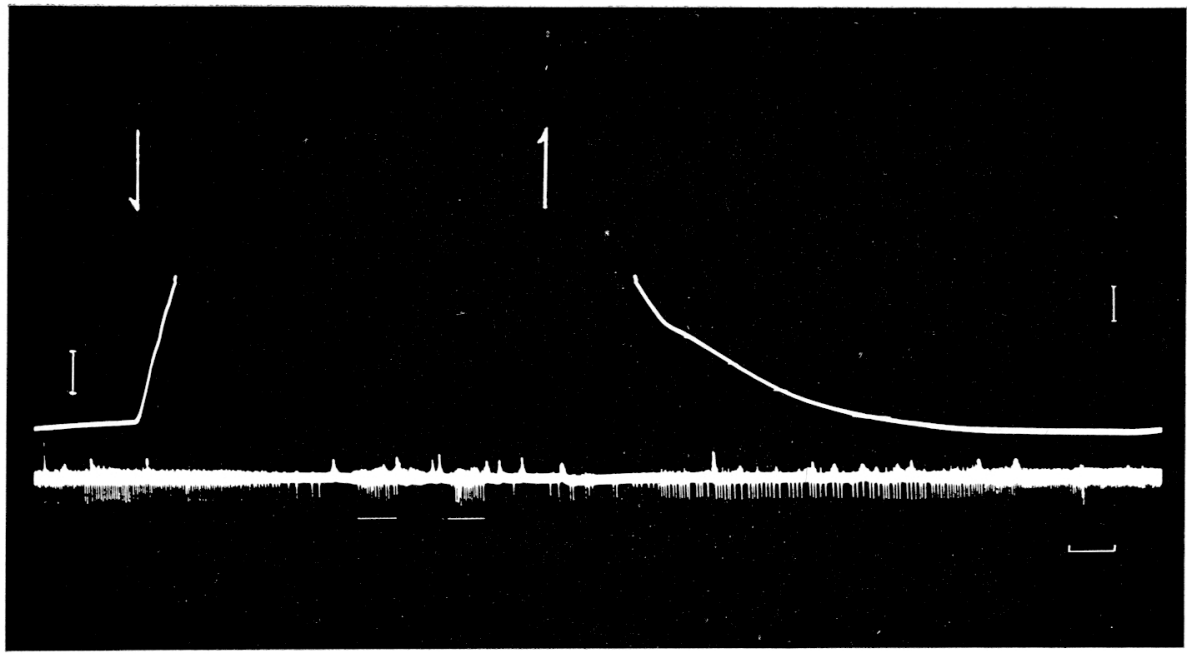

Fig. 7A. Effect of PUSN stimulation on sphincter activity inhibited by passive vesical distension. Top : intravesical pressure. Vertical arrows on the top indicate onset $(\downarrow)$ and finish (1) of vesical filling. Middle: sphincter discharge. Bottom: stimulation of PUSN. Voltage calibration $200 \mu \mathrm{V}$ (vertical line on the right), scale for intravesical pressure $10 \mathrm{~cm} \mathrm{H}_{2} \mathrm{O}$ (vertical line on the left) and time scale $2 \mathrm{sec}$. (horizontal line on the lower right).

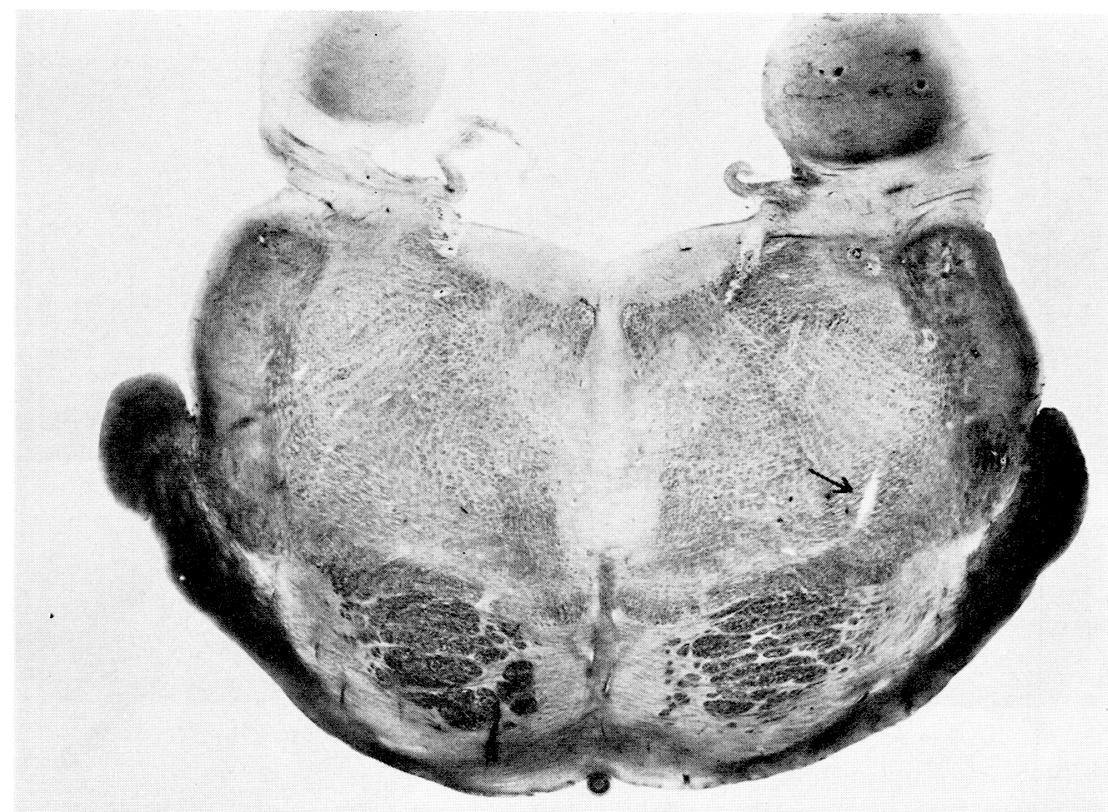

Fig. 7B. Electrolytic lesion made at the stimulated point of recording in FIG. 7A, Marked with arrow. 
distension, the sphincter discharge reappeared during PUSN stimulation. In this case, however, the discharge frequency was less than that in the resting state (FIG. 7).

Effect of PDN stimulation, PUSN stimulation or both on sphincter activity in bladderless preparation. Similar experiments were repeated in cats, the bladder of which had been removed without injuring external urethral sphincter and its nervous supply. On PDN stimulation in such a preparation the sphincter discharge promptly disappeared. On the other hand, the sphincter activity was enhanced during PUSN stimulation. Moreover, if the PUSN was stimulated for a while during the course of long-lasting PDN stimulation, the tonic discharges of the sphincter reappeared corresponding to the period of PUSN stimulation (FIG. 8). Thus, the mechanism of interference between the PUSN and the PDN was quite similar in the preparation either with or without the bladder.

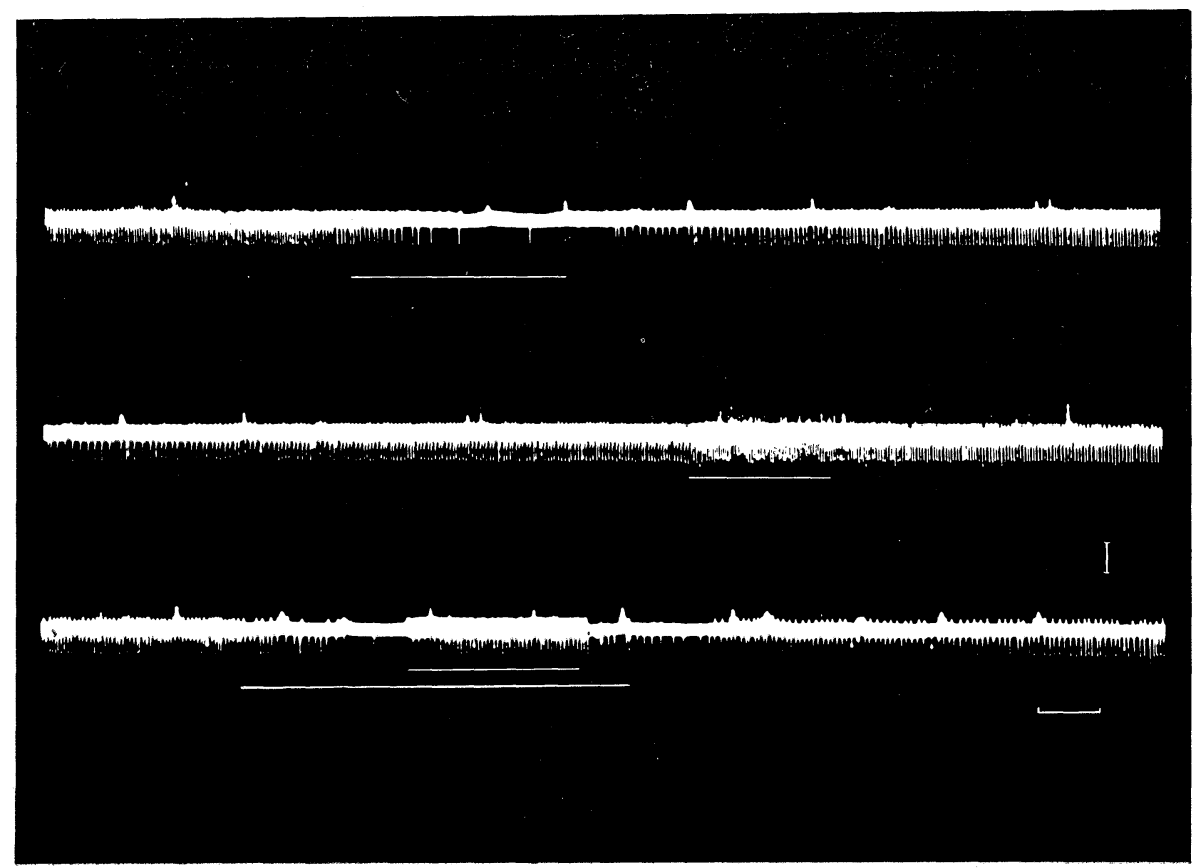

FIG. 8A. Responses of external urethral sphincter to PDN and PUSN stimulation on bladderless preparation. Upper section: sphincter response (upper) to PDN stimulation (lower). Middle section: sphincter response (upper) to PUSN stimulation (lower). Lower section: sphincter responses (upper) to simultaneous stimulation of PUSN (middle) and PDN (lower). Voltage calibration $200 \mu \mathrm{V}$ (vertical line on the right) and time scale $2 \mathrm{sec}$. (horizontal line on the lower right). 


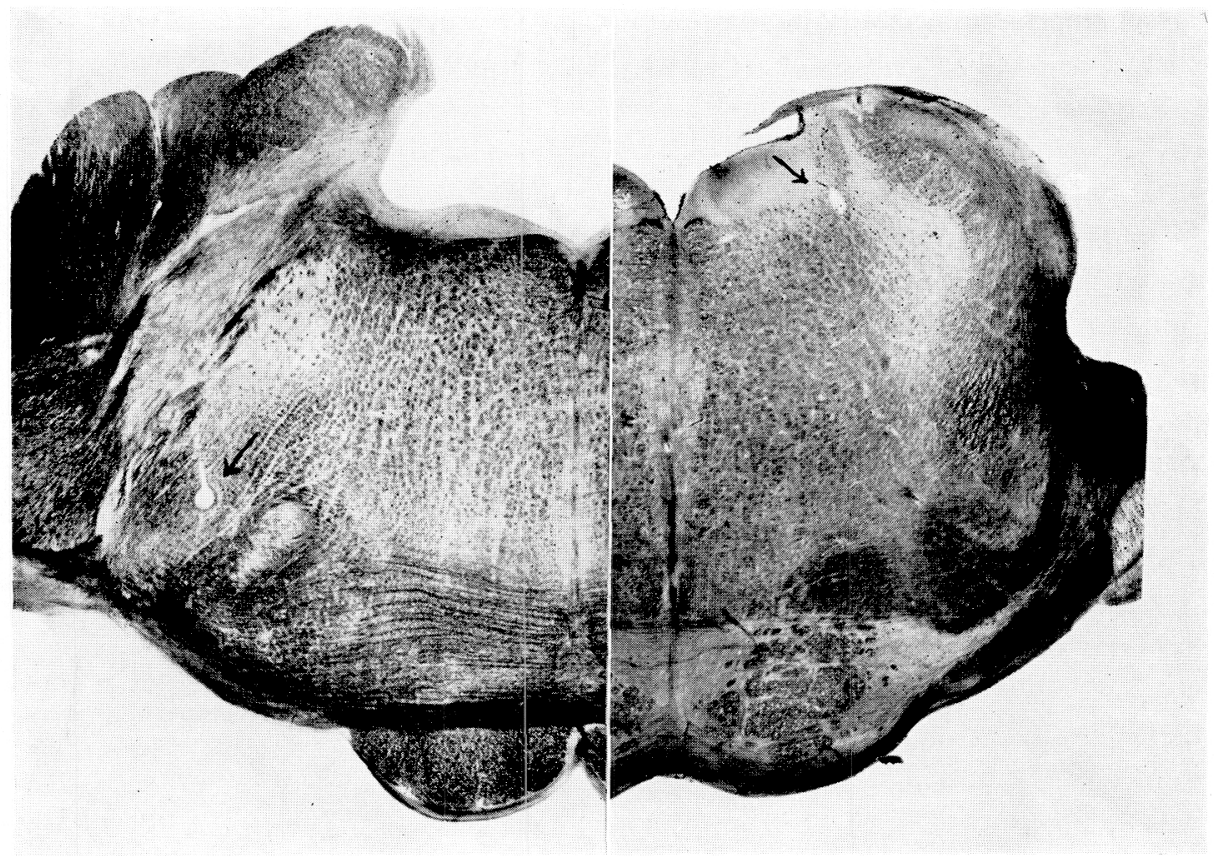

FIG. 8B. Electrolytic lesions made at the stimulated points of recording in FIG. 8A are indicated with arrows. Showing in the right half PDN and in the left half PUSN.

\section{DISCUSSION}

As GARRY et al. ${ }^{2)}$ had described, it was confirmed by the present series of experiments that either of the passive distension or the reflex contraction of the bladder inhibited the external urethral sphincter. Therefore, the decrease in sphincter activity subsequent to electrical stimulation to the PDN can also be related to the vesical contraction provoked by PDN stimulation. However, another inhibitory effect of PDN upon PUSN in the central nervous system must be considered, for the inhibitory effect of PDN stimulation upon the sphincter activity was clearly demonstrated in the bladderless preparations. There are two possibilities to explain this inhibitory effect; the inhibition was effected at the spinal level or at the pontine level. As was already described in detail elsewhere ${ }^{8)}$, the descending connections originating from the bulbar lateral nucleus (bulbar vesico-constrictor center), participating in the lateral reticulo-spinal tract, descend to the lumbo-sacral division of the cord. On the way, some of the relevant fibers gradually shift ventrally from the mid-thoracic level and terminate in the ventral horn cells of the lumbosacral segments. These fibers may possibly subserve inhibition of the external urethral sphincter in case of excitation of the bulbar vesico-constrictor center. 
Since the PDN of one side has bilateral connections to the bulbar vesicoconstrictor center ${ }^{11}$, PDN stimulation may possibly result in the inhibition of sphincter by this connection. However, it is more likely that the PDN gives the direct inhibitory effect on the PUSN, because it was confirmed that the PDN of one side send connections to PUSNs of both sides ${ }^{11}$.

On the other hand, the PUSN inhibits the PDN activity. As mentioned above, if during the prolonged stimulation of PDN, PUSN stimulation is superposed, the sphincter discharge, which was inhibited by PDN stimulation, reappears during PUSN stimulation. This phenomenon too, can be most readily explained by supposing a direct inhibitory effect of the PUSN on the $\mathrm{PDN}$, for the reappearance of the sphincter discharge was observed even in bladderless preparations. The relevant fiber connections between the PUSN and PDN were clearly demonstrated in the previous paper ${ }^{5)}$.

Urine storage and micturition are two main functions imposed on the urinary bladder. These two functions are reciprocal with each other. During storage of urine the bladder contraction is inhibited and the sphincter continence prevails. On the contrary, during micturition the bladder contraction vigorously continues and the sphincter relaxes. In the previous papers ${ }^{5,7)}$ it was demonstrated that PUSN stimulation provokes vesical relaxation at high intravesical pressure, in addition to enhancement of the sphincter activity. Present series of experiments clearly showed that the enhancement of sphincter activity resulted from PUSN stimulation even when vesical contraction was proceeding by PDN stimulation. This result may afford a clue for the elucidation of the mechanism of "cessation of voiding at will".

Most of the investigators are in agreement to ascribe the initial stimulus to reflex micturition to the attainment of a critical degree of bladder filling ${ }^{4}$. As described by GARRY et al. ${ }^{2)}$ and confirmed in the present experiments, the rise in the intravesical pressure markedly inhibits the sphincter activity. However, simultaneously, the increase in intravesical pressure facilitates the excitation of the bulbar vesico-relaxer center and inhibits the activity of the vesico-constrictor centers ${ }^{9)}$. To circumvent this repugnancy, certain suprabulbar control system is indispensable. There is an area in the mesencephalon, stimulation to which markedly facilitates vesical contraction ${ }^{3)}$. This area has strong fiber connections with the bulbar vesico-relaxer areas on both sides ${ }^{10}$. These connections are thought to subserve the release of the bulbar and spinal vesico-constrictor centers from vesico-relaxer effect due to the increased intravesical pressure. In addition to the mesencephalic control system, there is a pontine control system as demonstrated in the present experiment. The excitation of PDN inhibits markedly the PUSN activity, thus indirectly liberating the bulbar vesico-relaxer center from the facilitatory effect of the PUSN. Under the control of suprabulbar system the vesical contraction could smoothly proceed against the increase in intravesical pressure. Thus, the PDN excita- 
tion results in a multiplication of vesico-constrictor effect ${ }^{4)}$, for as described in detail in the previous paper ${ }^{11}$, the PDN of one side send bilateral connections both to the bulbar and sacral vesico-constrictor centers. BARRINGTON's ${ }^{1)}$ historical discovery that bilateral destruction of the dorsolateral reticular formation of the rostral pons results constantly in a permanent inability to void completely, has been thus pertinently explained.

\section{SUMMARY AND CONCLUSION}

In the previous papers ${ }^{11,7,5)}$ two antagonistic areas in the pons concerning micturition, the pontine detrusor nucleus (PDN) and the pontine urine-storage nucleus (PUSN), were reported. In the present paper the interference between these two pontine areas was investigated by means of electromyogram of the external urethral sphincter. The conclusions are as follows:

1. PDN stimulation elicits strong vesical contraction with simultaneous disappearance of discharge from the external urethral sphincter.

2. In the resting bladder, stimulation of PUSN results in an increase of discharge from the external urethral sphincter despite the absence of recognizable change in the intravesical pressure.

3. If during the long-lasting PDN stimulation PUSN is stimulated for a while, sphincter discharge reappears with the inhibition of the rise of the intravesical pressure corresponding to the period of PUSN stimulation. This phenomenon can be observed also in bladderless preparations.

4. The significance of this interference for micturition and urine-storage was discussed in detail.

\section{REFERENCES}

1) BARrington, F. J.F. The effect of lesions of the hind-and midbrain on micturition in the cat. Quart. J. Exptl. Physiol. 15: 81-102, 1925.

2) Garry, R.C., T.M. Roberts and J.K. Todd. Reflexes involving the external urethral sphincter in the cat. J. Physiol. 149: 653-665, 1959.

3) Koyama, Y., A. Makuya And M. Kuru. Vesico-motor areas in the cat midbrain. Japan. J. Physiol. 12: 63-80, 1962.

4) Kuru, M. Nervous control of micturition. Physiol. Rev. 45: 452-494, 1965.

5) Kuru, M. And T. Iwanaga. Ponto-sacral connections in the medial reticulospinal tract subserving storage of urine. (To appear in J. Comp. Neurol.)

6) Kuru, M., Y. Koyama and T. Kurati. The bulbar vesico-relaxer center and the bulbo-sacral connections arising from it. A study of the function of the ventral reticulo-spinal tract. J. Comp. Neurol. 115: 15-26, 1960.

7) Kuru, M., Y. Koyama and H. Ozaki. Part of the brain stem controlling the tone of the external urethral sphincter. Proc. Jap. Acad. 39: 530-533, 1963.

8) Kuru, M., T. Kurati and Y. Koyama. The bulbar vesico-constrictor center and the bulbo-sacral connections arising from it. A study of the function of the lateral reticulo-spinal tract. J. Comp. Neurol. 113:365-388, 1959. 
9) Kuru, M., H. Ozaki AND T. Kurati. Effect of simultaneous stimulations of bulbar vesico-constrictor and vesico-relaxer centers. J. Comp. Neurol. 116: 195208, 1961.

10) Kuru, M., A. Makuya and Y. Koyama. Fiber connections betweer the mesencephalic micturition facilitatory area and the bulbar vesico-motor centers. $J$. Comp. Neurol. 117 : 161-178, 1961.

11) Kuru, M. AND H. Yamamoto. Fiber connections of the pontine detrusor nucleus (Barrington). J. Comp. Neurol. 123: 161-185, 1964. 